\title{
General Relativistic MHD Simulations of Jets from Accreting Black Holes
}

\author{
Julian Krolik* \\ Johns Hopkins University, Baltimore, USA \\ E-mail: jhk@pha.jhu.edu \\ John Hawley \\ University of Virginia, Charlottesville USA \\ E-mail: jh8hevirginia.edu
}

\begin{abstract}
Most models of jet launching from black holes have concentrated on the combined effects of large-scale magnetic fields and rotation, where the rotation may be either by orbiting matter or by a spinning black hole. We use large-scale general relativistic 3-d MHD numerical simulations to investigate the efficacy of these models, restricting ourselves to magnetic fields that are initially confined within the accreting matter. When the initial field geometry is dipolar, the field spontaneously extends to large scales within a cone centered on the rotation axis. If the black hole spins rapidly, a strong Poynting flux jet is then created, with luminosity comparable to that likely to be generated by photon radiation from the accretion disk. Other initial magnetic field geometries (e.g., quadrupolar) can produce much weaker jets. A slower matter-dominated outflow, expelled by pressure gradients in the disk corona, can also form along the outside of the cone containing the Poynting flux jet.
\end{abstract}

VI Microquasar Workshop: Microquasars and Beyond September 18-22, 2006

Como, Italy

\footnotetext{
${ }^{*}$ Speaker.
} 


\section{Background}

\subsection{Magnetic fields and rotation: the recipe of choice}

For many years, the favorite scheme of nearly every theorist for launching jets from accretion flows has been somehow to combine large-scale magnetic fields and rotation. There are several reasons why this approach has been so popular. To start with, magnetic fields are nearly ubiquitous in astrophysics, and the high electrical conductivity that guarantees flux-freezing is almost as common as the magnetic fields themselves. Dynamical coupling between plasma and magnetic fields almost doesn't rank as an assumption-it's to be expected.

Another reason for the continuing focus on this framework is that jets require large-scale coherence. The material inside them travels on more or less straight lines for long distances, expanding sideways remarkably little (in angular terms) even as it traverses many orders of magnitude contrast in linear scale. Magnetic fields offer both a natural source of large-scale connections and, in the form of properties like hoop stress, a potential avenue for creating collimation (although whether magnetic hoop stress can really do the job is a matter of considerable controversy; for a sampling of recent views, see $[14,11,18,7,19])$.

Rotation is also of help here. Gravity on its own defines no special direction. Rotation, however, takes place around an axis. The orientation of this axis then provides a natural choice for breaking the isotropy of gravity and selecting the direction for the jet. Rotation also has several useful dynamical consequences. For example, centrifugal barriers can help keep unwanted matter out of the region around the axis, preserving that region as a low-inertia domain.

Perhaps the strongest elements that make this pair so attractive, though, are two specific dynamical models, both originating with Roger Blandford and collaborators: one known as BlandfordZnajek [2], the other known as Blandford-Payne [3]. Although both involve non-trivial assumptions, these assumptions are plausible enough that many believe they are likely to obtain in real situations.

\subsection{The Blandford-Znajek model}

In the former model, a large-scale poloidal magnetic field is supposed to thread the event horizon of a rotating black hole. Its intensity is so great and the local inertia is so small that it is "force-free", i.e., magnetic field dynamics are controlled entirely by minimizing the field energy subject to the topological constraints imposed by $\nabla \cdot \vec{B}=0$, without any effect due to the matter's inertia. The only role of the matter is to provide the electrical currents necessary to sustain the magnetic field. Rotation enters through the rotation of the black hole itself, and does so in two ways: the frame-dragging it imposes on nearby space-time, when acting on poloidal magnetic field, enables Poynting flux; and the rotational kinetic energy of the black hole (the difference between its mass $M$ and its irreducible mass $\left.M_{i r}=M\left[\left(1+\sqrt{1-a_{*}}\right) / 2\right]^{1 / 2}\right)$ provides a source of energy even in the absence of ongoing accretion. Here we adopt an abbreviated notation for the black hole spin parameter, setting $a / M \equiv a_{*}$.

One way to view the effect of the frame-dragging is that it creates an effective electric field in almost any observer's frame (Fig. 1). Special relativistically, if the electric field is zero in the fluid frame (i.e., if the MHD approximation applies because there is enough mobile charge to 

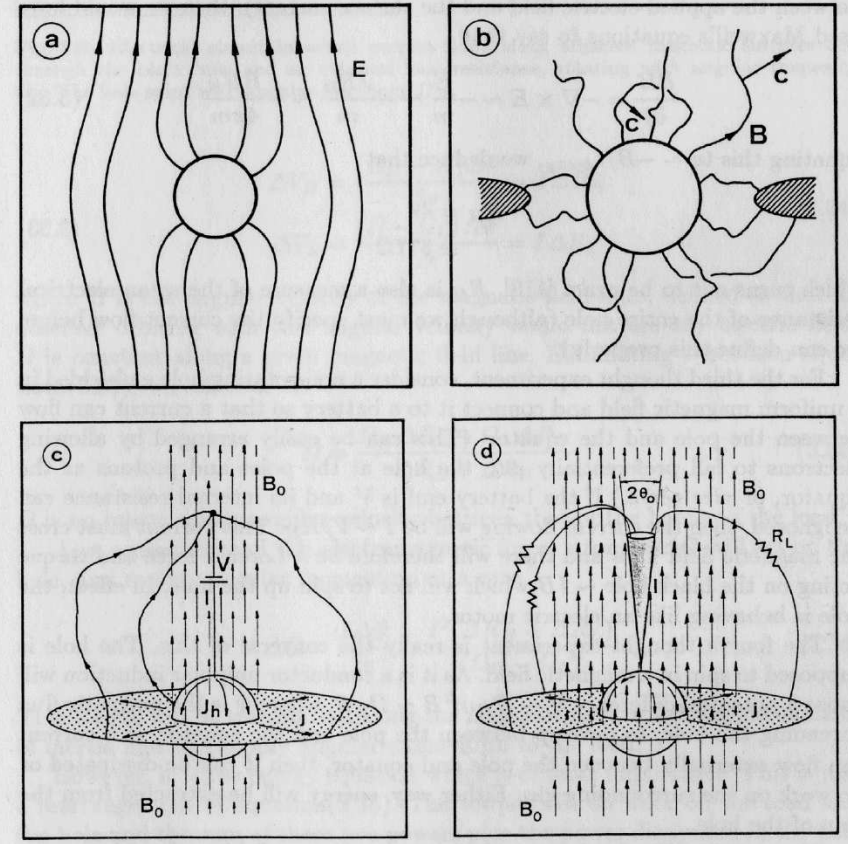

Figure 1: A four-panel montage taken from reference [17]. Field that is initially purely poloidal (upper-left) through the action of space-time rotation (lower-left) ends up driving currents that can be described in terms of a lumped parameter model in which a schematic load at infinity of resistance $R_{L}$ in combination with the resistance of the horizon determines the net current (lower-right).

cancel any electric field), in frames whose velocities differ by $\vec{v}$, there is an electric field $\vec{E}=$ $-\vec{v} / c \times \vec{B}$. This effect, although quantitatively slightly modified, persists in general relativity. It is of special importance when the black hole rotates because there is a net rotational difference even between non-rotating observers at infinity and fluid with zero angular momentum in the ergosphere. Currents driven by this electric field deep within the ergosphere can cross field lines because of the effective resistivity of the event horizon; when these current loops close at infinity after having travelled out along one field line and back along another, they can deliver power to long distance.

Another way to view this story is to note that even magnetic fields that are radial at infinity acquire a transverse component in the ergosphere as they are forced to rotate. Transverse timevarying magnetic field leads directly to Poynting flux, which can, of course, carry energy to infinity.

\subsection{The Blandford-Payne model}

Pure Newtonian physics suffices to drive axially-oriented outflows in the disk-wind picture of Blandford and Payne. If magnetic field traces its way from large distances down through the disk and back out again (as illustrated in Fig. 2), its energy density at sufficiently large distance from the disk must dominate that of any associated matter. In that sense, the magnetic field is "stiff" at large distances from the disk. Because the field lines are also attached firmly to the orbiting matter in the disk, they must rotate with the orbital frequency of their attachment points. Where the fieldlines become stiff, they force the matter to rotate this fast, too. Provided the fieldlines rake back at a sufficiently sharp angle, the cylindrical radius in these distant regions is large enough relative to the 


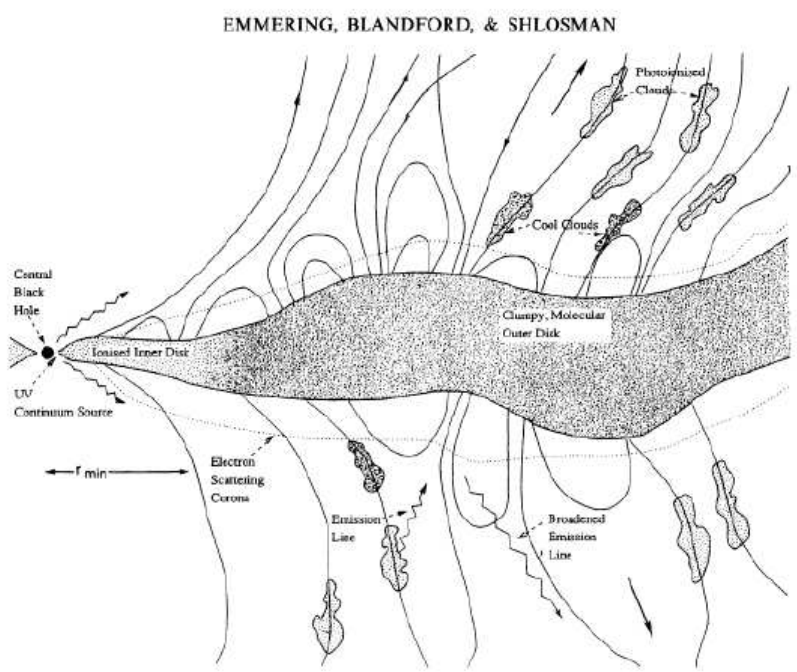

Figure 2: A generic disk-wind, as envisioned by Emmering et al. [5]. Some magnetic field loops emerge from the surface of the disk, only to close within the disk corona. However, others connect the disk to infinity. Streams of fluid are expelled to large distance along those open field lines.

radius of the attachment points that the angular momentum acquired by the matter is larger than the angular momentum whose contribution to the effective potential balances gravity. If so, there is a net outward force on the matter. Pushed outward along the fieldlines and compelled to rotate with them, the matter's angular momentum increases still further, and a wind can be driven.

\subsection{Questions}

Although these two models are plausible enough, they raise many questions. It is our aim, of course, to try to answer them. In order to motivate the results presented later in this talk, it is useful to lay out a number of the most pressing of them here.

Both the Blandford-Znajek and Blandford-Payne models posit rather specific magnetic field structures and boundary conditions; can these be realized in Nature? Will accretion mechanics result in a field attached to the black hole strong enough to be force-free? If field is brought to the black hole in the accretion flow, will net flux remain after reconnection snaps off loops? The dramatic compression that takes place in accretion onto a black hole from any imaginable source will forcibly bring close together oppositely-directed field segments-how much flux can survive all the way to the event horizon?

In fact, although the standard forms of both models require net magnetic flux, is that a necessary assumption? Perhaps the accretion flow itself can generate enough large-scale magnetic field structure to do the job.

Which of the two models is most important when? Some [16] have argued that Poynting flux from the disk must inevitably overwhelm any imaginable Poynting flux from the black hole, but their argument assumed that the field near the event horizon would be at most comparable in strength to the field in the disk - is that a reasonable assumption? They similarly argued that most of the energy in the outflow would have to be derived from accretion rather than from the rotational kinetic energy of the black hole—can these two sources be cleanly separated? 


\section{Overview of Simulations and their Results}

Following the behest of Occam's Razor, let's try the simplest model first: We know that jets are common in sources where active accretion onto a black hole is taking place, so we will begin by asking whether jets can arise as a result of accretion. For some time now [1], it has been understood that the angular momentum transport that is essential to accretion is largely accomplished by correlated MHD turbulence, driven by an underlying magneto-rotational instability. The only mathematical tool we possess that can describe fully nonlinear turbulence is numerical simulation; perforce, that is therefore the tool we must employ.

After many years of development, the computer codes available for numerical simulations of this problem have become quite sophisticated, although a number of gaps and difficulties still remain. In the code upon whose results we will primarily lean [4], dynamics are computed in terms of the ideal MHD approximation in 3(+1) dimensions and full general relativity. Other codes of similar capability have also been developed $[6,10,13]$, although some of these have mostly been used on problems with axisymmetry. For the particular simulations reported here [8], the computational volume was a quarter-sphere centered on the black hole that stretches out to a radius of $120 M$ (here and following, we set $G=c=1$ ), but whose azimuthal range runs only from 0 to $\pi / 2$.

The principal limitation of this code lies on the side of thermodynamics. It assumes that fluid elements evolve adiabatically (with $\gamma=5 / 3$, appropriate to an ideal mono-atomic gas that is nonrelativistic in the local fluid frame) except in the vicinity of shocks, where an artificial bulk viscosity is introduced in order to properly capture entropy production and curb numerical "ringing". Thus, any heat that is liberated either by resistive dissipation of magnetic field or physical viscosity acting on fluid motions is ignored. Heat lost by radiation goes likewise unaccounted. Because our aim is to focus on questions having primarily to do with inflow and outflow dynamics driven by magnetic forces, we regard this limitation as tolerable for the time being.

The initial condition in all of our simulations was a hydrostatic axisymmetric torus of gas, with central pressure maximum placed at $r=25 M$ in the equatorial plane. The specific entropy of the gas was uniform, and large enough to give the torus an aspect ratio $H / R \simeq 0.1-0.15$, for vertical density scale-height $H$. We begin with all the mass on the computational grid so that no mass is brought in across the outer boundary-to do otherwise would invite the introduction of numerical noise. A hydrostatic configuration provides a quiet start, without violent transients. The design disk-thickness was chosen so as to be reasonably thin, but still thick enough that we could construct a grid that would resolve it.

Some, but not all, of the magnetic properties were similarly uniform throughout our simulations. The mean plasma $\beta$, the ratio between gas pressure and magnetic pressure, was set to 100 at the start, and in all of our simulations to date, the magnetic field closed entirely within the matter, so that there was no net magnetic flux. All initial field configurations were also axisymmetric.

The simulations differ, however, in the detailed geometry of the initial magnetic field. In most of the cases we have studied, the field was purely poloidal, and was arranged in an initial configuration we call "dipolar": the fieldlines follow the contours of density in the hydrostatic torus, so that they wrap around the density maximum, which is located in the equatorial plane. In others, however, the fieldlines in the initial state formed a pair of nested poloidal loops, one centered 

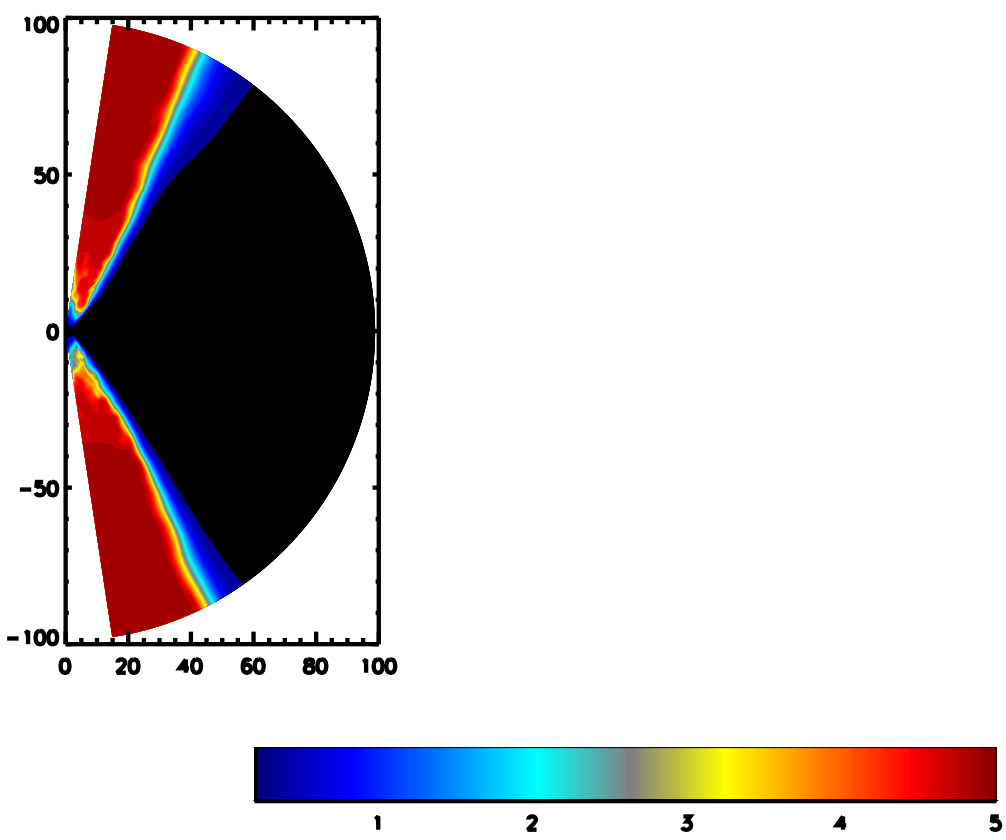

Figure 3: Mass-weighted and azimuthally-averaged $u^{r}$ in a snapshot taken from a simulation with $a_{*}=0.9$.

above the equatorial plane, the other centered symmetrically below. These we call "quadrupolar".

In addition to varying the magnetic field geometry, we have also experimented with varying the black hole spin parameter $a_{*}$. We have now performed simulations with $a_{*}=-0.9,0.0,0.5$, $0.9,0.93,0.95,0.99$, and 0.998 . Here a positive sign means that the accreting matter orbits in the same sense as the black hole spins, while a negative sign means that, although the matter orbits in the equatorial plane of the black hole rotation, it revolves in the opposite sense.

With respect to accretion properties, there are only quantitative differences between dipolar and quadrupolar initial magnetic geometries. However, they differ sharply in regard to outflows. To zeroth order, the distinction is simple: when the field is dipolar and $a_{*} \neq 0$, a strong jet forms spontaneously; when either one of these conditions does not apply, any outflow is far weaker.

All strong jets, at least within this set, bear a strong resemblance to one another, contrasting primarily in their strength. Filling a cone surrounding the rotation axis, we find a magneticallydominated plasma of very low density that moves outward at relativistic speeds, as shown in Figure 3. Forming a sheath around the outside of this relativistic jet, there is a matter-dominated outflow whose radial speed is only mildly relativistic; as shown in the figure, the radial component of the four-velocity $u^{\mu}$ tapers off rapidly with increasing polar angle at the edge of the outflow funnel. The remainder of this talk will be devoted to describing these two jet components in greater detail.

\section{The Magnetically-Dominated Filled Cone}

Although no large-scale magnetic field is present at the start of the simulations, when the field's initial configuration is dipolar, one is always created soon after the beginning of the simulation. The 

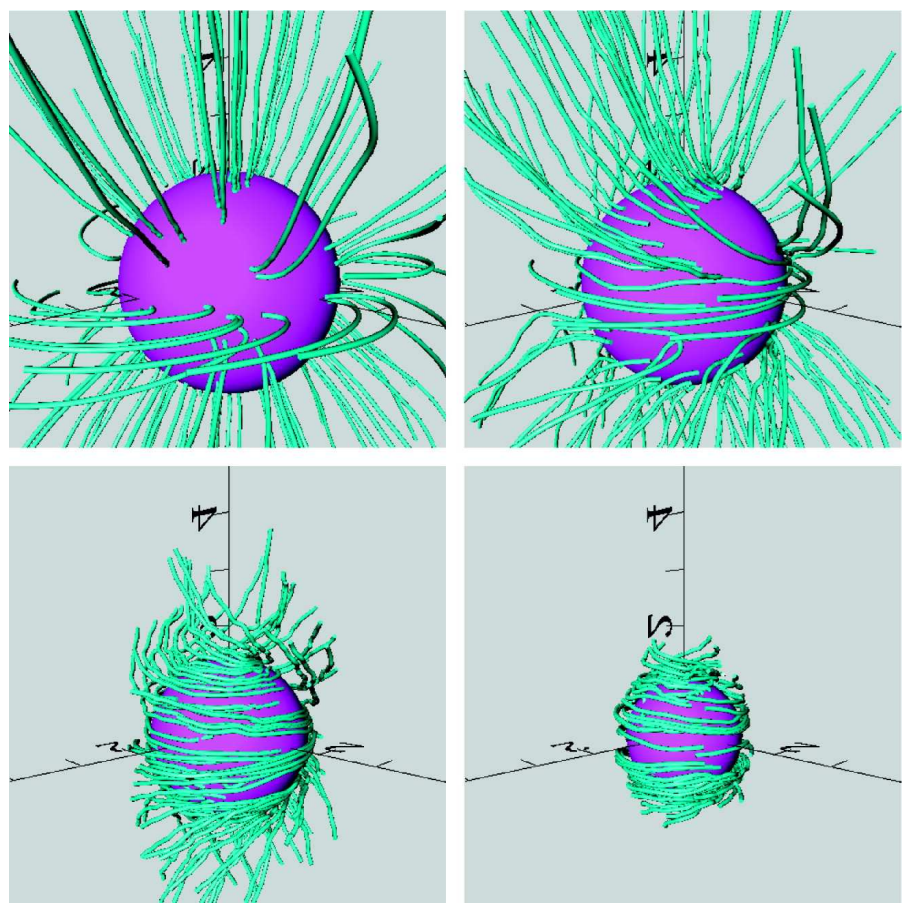

Figure 4: Fieldlines near the black hole [9] in four simulations differing only in black hole spin: $a_{*}=0$ (upper left); $a_{*}=0.5$ (upper right); $a_{*}=0.9$ (lower left); and $a_{*}=0.998$ (lower right). Observe how the field, nearly radial near the rotation axis in the Schwarzschild case, wraps tighter and tighter as the black hole spins faster and faster.

magneto-rotational instability grows at a rate $\sim \Omega$, so by the time one orbital period at $r \sim 20 M$ has passed (i.e., 500-700M), a thin "foot" of material has begun to move rapidly inward, stretching the field radially as it does so. Shear creates toroidal field from radial at the local rotation rate. Although the matter mostly stays close to the equatorial plane, because there is neither field nor matter much above or below the plane, there is a strong vertical $\nabla B^{2}$ force and the field puffs up. Once the accretion flow enters the plunging region inside the innermost stable circular orbit (ISCO), matter begins to drain rapidly along the radial component of the field toward the event horizon. Shorn of its inertia, the leading edge of the poloidal field loops that have been brought in close to the black hole inflate along both the top and bottom ends of the rotation axis. By $\sim 1000 \mathrm{M}$ after the beginning of the simulation, a sizable amount of flux has been attached to the surface of the black hole, and the associated fieldlines have streamed out all the way to the outer radial boundaries of the axial cone.

When all this takes place in the context of a Schwarzschild $\left(a_{*}=0\right)$ spacetime, the field in the axial cone is almost purely radial, as shown in the first frame of Figure 4. However, if the black hole rotates at all, frame-dragging close to the event horizon pulls the field into a helix, imprinting a transverse magnetic field component at the base of the outflow. That black hole rotation is the key to the field's twist is confirmed by two facts: its absence in the Schwarzschild case; and its sensealways with the rotation of the black hole, even when the accreting matter orbits the opposite way.

Although fieldline rotation rate is a fuzzy concept when the field is neither axisymmetric nor 


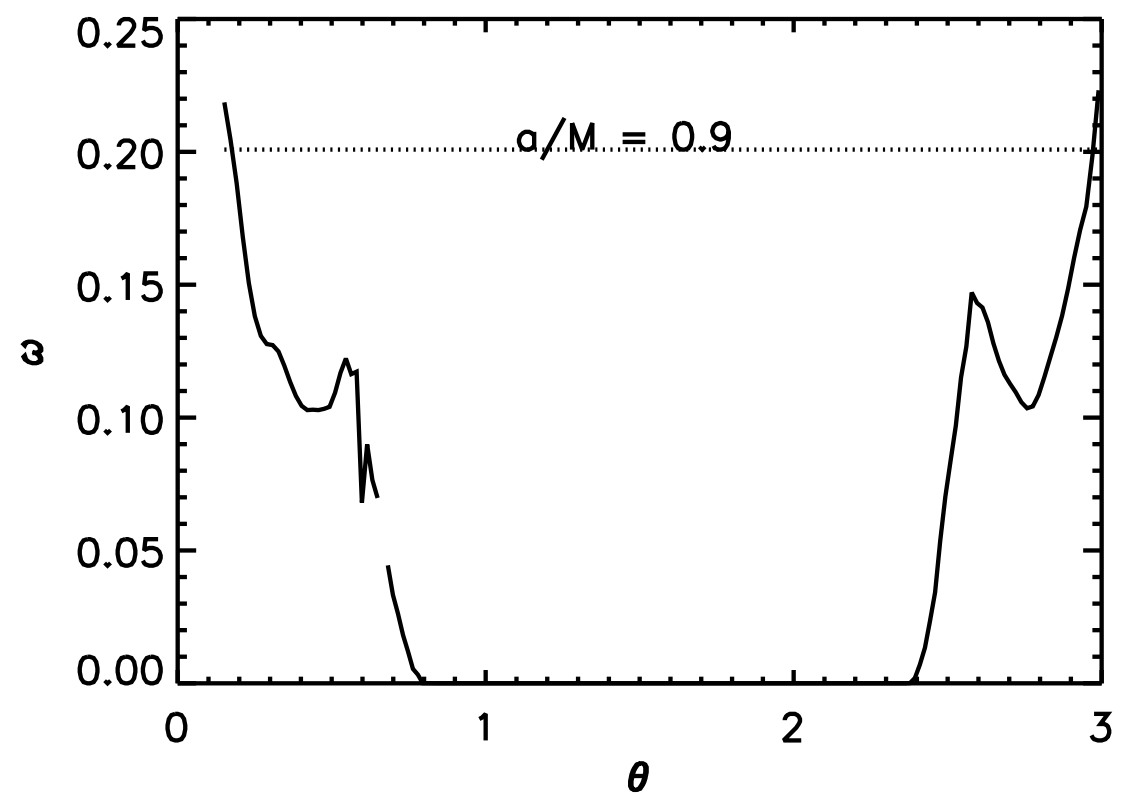

Figure 5: Fieldline rotation rate in the outflow as a function of polar angle for a simulation with $a_{*}=0.9$ (solid curve). The classical Blandford-Znajek model prediction is shown by the dotted line.

time-steady, we can define an approximate version for empirical study:

$$
\omega \equiv V^{\phi}-\mathscr{B}^{\phi} \frac{V^{r} \mathscr{B}^{r} g_{r r}+V^{\theta} \mathscr{B}^{\theta} g_{\theta \theta}}{\left(\mathscr{B}^{r}\right)^{2} g_{r r}+\left(\mathscr{B}^{\theta}\right)^{2} g_{\theta \theta}}
$$

Here $\mathscr{B}^{i}=[i j k] F_{j k}$, the magnetic field as it enters into the Maxwell tensor $F_{\mu v} ; V^{i}=u^{i} / u^{t}$ is the spatial "coordinate velocity"; and $g_{\mu \nu}$ is the Kerr metric tensor. The second term describes how fast matter slips along the toroidal field relative to the rotation of the poloidal part. In the time-steady axisymmetric limit, equation 3.1 reduces to the standard definition of fieldline rotation (as found, e.g., in [15]).

In the simplest form of the classical Blandford-Znajek model, which is both axisymmetric and time-steady, the fieldlines are all well-defined and are predicted to rotate at exactly half the rotation rate of the black hole, $\omega_{H}=0.5 a_{*} /\left(1+\sqrt{1-a_{*}^{2}}\right)$. Because our fieldlines do not follow paths that are known a priori, and can, in fact, be expected to wander a bit, we must adopt an approximate ansatz in order to transform our local definition of $\omega$ to one that applies globally to an entire fieldline. Consistent with our finding that the magnetically-dominated outflow has walls that are roughly conical, we choose to average $\omega$ along lines of fixed polar angle. With that definition, we find that the rotation rate can sometimes be as large as $\omega_{H} / 2$, but is often factors of a few smaller (Fig. 5). In their axisymmetric study, McKinney and Gammie [12] found a similar result, although the discrepancy between their fieldline rotation rate and $\Omega_{H} / 2$ was somewhat smaller than ours.

As promised, the transverse magnetic field created at the base of the flow by rotational framedragging leads to significant Poynting flux (Fig. 6). Although it is highly non-uniform over the 


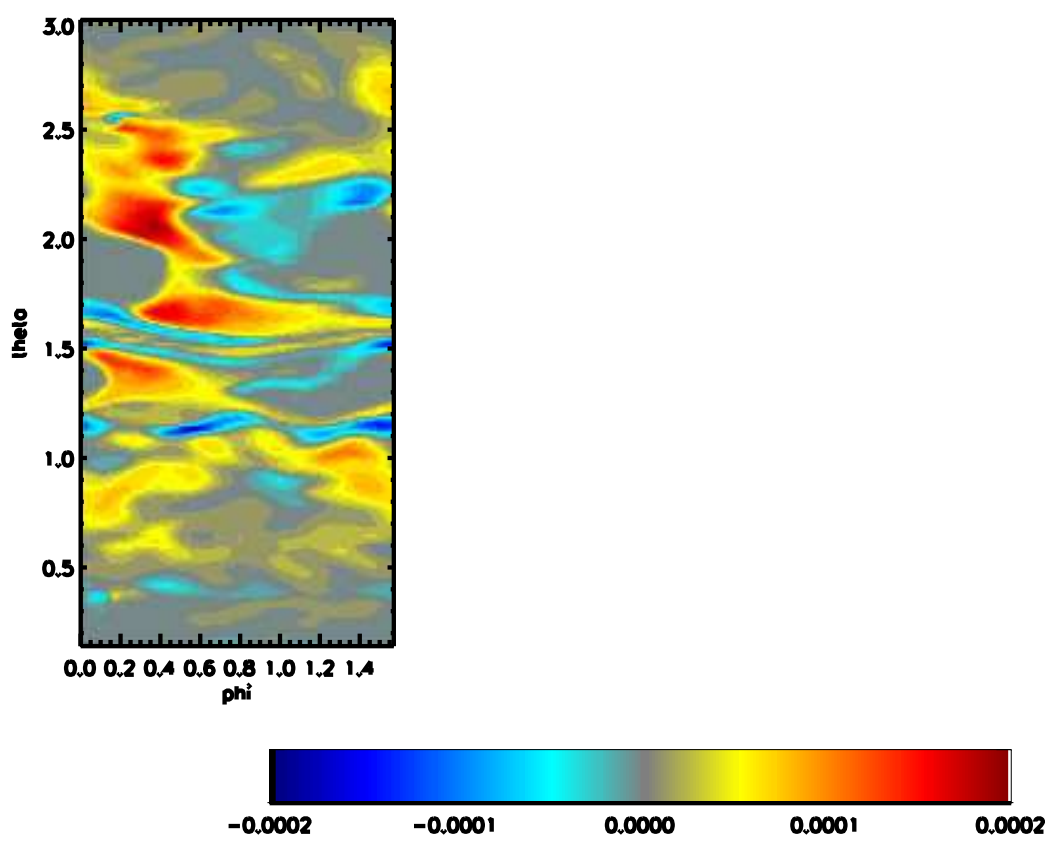

Figure 6: Instantaneous Poynting flux on the inner boundary (immediately outside the event horizon) in a simulation with $a_{*}=0.9$.

surface of the event horizon, including fluctuations in sign, there is a significant net outward flow of electro-magnetic energy in all initially dipolar simulations of a rotating black hole.

One might raise the question of how a black hole can emit energy, as all photons are trapped by the event horizon. The answer is that in the ergosphere, which permits particle orbits having negative energy-at-infinity, there can also be fluid elements whose electromagnetic energy-at-infinity is negative. The key ingredient is for these fluid elements to be rotating sufficiently rapidly in the direction opposite to the black hole's spin. If these fluid elements are captured by the black hole, they deliver a flux of negative energy, a process equivalent to the black hole losing positive energy.

The magnitude of the Poynting flux in the jet can be quite substantial. It is most convenient to measure this Poynting flux in the natural units of the problem, the rate at which rest-mass energy is accreted by the black hole; that is, we will quote "efficiencies" $\eta_{\mathrm{EM}}$ for the Poynting flux that have the same meaning as the traditional radiation efficiency of the accretion disk. In fact, in Table 1 we use the radiative efficiency of the Novikov-Thorne model as a standard of comparison.

As can be readily seen by study of this table, the Poynting flux efficiency is a stronglyincreasing function of black hole spin. Negligible at low spin, at high spin the Poynting flux can contribute to the total system output at a level comparable to that provided by radiation from the accretion disk. Because the Poynting flux is intimately associated with black hole rotation, its energy source can be thought of as the rotational kinetic energy of the black hole.

When thinking about the implications of Table 1, it is important to bear in mind that all the $\eta_{\mathrm{EM}}$ shown were computed from simulations in which the initial magnetic field was dipolar in geometry. We have explored two instances of quadrupolar geometry, $a_{*}=0$ and $a_{*}=0.9$. There is, of course, essentially no electromagnetic jet in the quadrupolar Schwarzschild case, just as for the 


\begin{tabular}{|c|c|c|}
\hline$a_{*}$ & $\eta_{\mathrm{NT}}$ & $\eta_{\mathrm{EM}}$ \\
\hline-0.9 & 0.039 & 0.023 \\
0.00 & 0.057 & 0.0003 \\
0.5 & 0.081 & 0.0063 \\
0.9 & 0.16 & 0.046 \\
0.93 & 0.17 & 0.038 \\
0.95 & 0.18 & 0.072 \\
0.99 & 0.26 & 0.21 \\
\hline
\end{tabular}

Table 1: Contrast as a function of black hole spin $a_{*}$ between time-averaged Poynting flux efficiency in rest-mass units $\eta_{\mathrm{EM}}$ and Novikov-Thorne accretion radiative efficiency $\eta_{\mathrm{NT}}$.

case of initially dipolar field accreted through a Schwarzschild metric. More strikingly, the timeaveraged Poynting flux in the quadrupolar simulation with a spinning black hole is approximately two orders of magnitude weaker than when the initial field was dipolar.

Clearly, the strength of the Poynting flux jet can depend greatly on the original field geometry. We do not know, of course, what the intrinsic geometry is in real sources. It is possible that some of the contrasts in observed jet strength from one jet to another or from time to time within a single source could have to do with the character of the field embedded in the accretion flow. The influence of field geometry on jet strength and the implications of that influence for observed sources are topics we intend to explore more fully in the near future.

\section{The Matter-Dominated Funnel-Wall Outflow}

As shown earlier, the speed of the outflow along the wall of the magnetically-dominated cone is considerably slower than the speed of the electromagnetically-dominated core. In a mass-weighted sense, $\left\langle u^{r}\right\rangle$ in the funnel-wall is generally only a few tenths of $c$. The funnel-wall outflow is also fundamentally unlike the magnetically-dominated jet in the funnel interior in that its properties are controlled by the accretion flow, rather than by the black hole.

For example, the mass-weighted and azimuthally-averaged specific angular momentum of the funnel-wall outflow $\left\langle u_{\phi}\right\rangle$ is consistently close to the specific angular momentum of the ISCO, both in sign and in magnitude, a few $M$ (Fig. 7). Moreover, $\left\langle u_{\phi}\right\rangle$ is also very nearly constant as a function of radius along the funnel wall. In this respect, the funnel-wall outflow is clearly not a BlandfordPayne wind because an essential part of Blandford-Payne dynamics is the torque exerted on the outflow by the rotating magnetic field, yet the angular momentum in this outflow hardly varies from place to place. It would also be fair to point out that because we exclude any initial largescale magnetic flux threading the disk, we would be able to see outflows of the Blandford-Payne variety only if there were a spontaneously-generated mechanism capable of expelling field loops from the disk all the way to the outer boundary.

The fact that the matter-dominated outflow has nearly constant angular momentum allows us to describe it in terms of an effective potential parameterized by that value of $\left\langle u_{\phi}\right\rangle$. The shape of this equipotential is illustrated in Figure 8. As this figure shows, the equipotential influences both the magnetically-dominated funnel interior and its matter-dominated sheath. It excludes matter from 


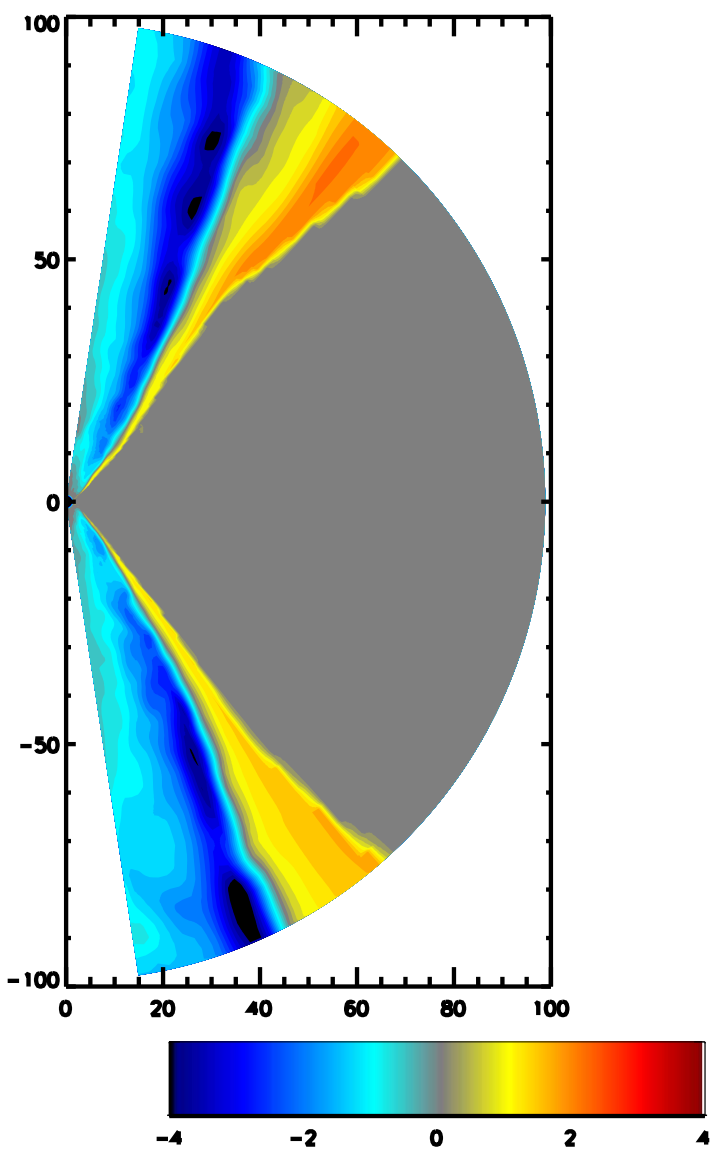

Figure 7: Instantaneous $\left\langle u_{\phi}\right\rangle$ in the outflow in a simulation with $a_{*}=0.9$.

the cone interior, allowing the rotating magnetic flux to dominate conditions there. Centrifugal exclusion also keeps the inertia in the funnel interior very low, so it's easy for magnetic forces to drive highly relativistic motion. In addition, the equipotential guides the matter-dominated part of the outflow: it travels out along the lowest equipotential surface connecting the inner accretion flow to infinity. That is, of course, exactly what one would expect from a hydrodynamic flow that has somehow been given enough energy to reach that level in the equipotential.

The question that then arises is: how does matter reach that energy? In the conditions of these simulations, the answer is, "by rising into the corona and being pushed out from there". The nature of our simulation makes it difficult to trace the energetics of fluid elements. We can, though, clearly identify the momentum flux in the matter-dominated outflow with the coronal gradients of gas pressure and magnetic pressure. At the same time, we must stress that the assumptions embedded in these simulations are not at all innocuous in the context of anything as intimately tied to the gas pressure as the matter-dominated outflow. Our very incomplete treatment of thermodynamics surely influences the pressure of the corona. For this reason, quantitative predictions about the strength of matter-dominated outflows in real systems must await simulations with additional 


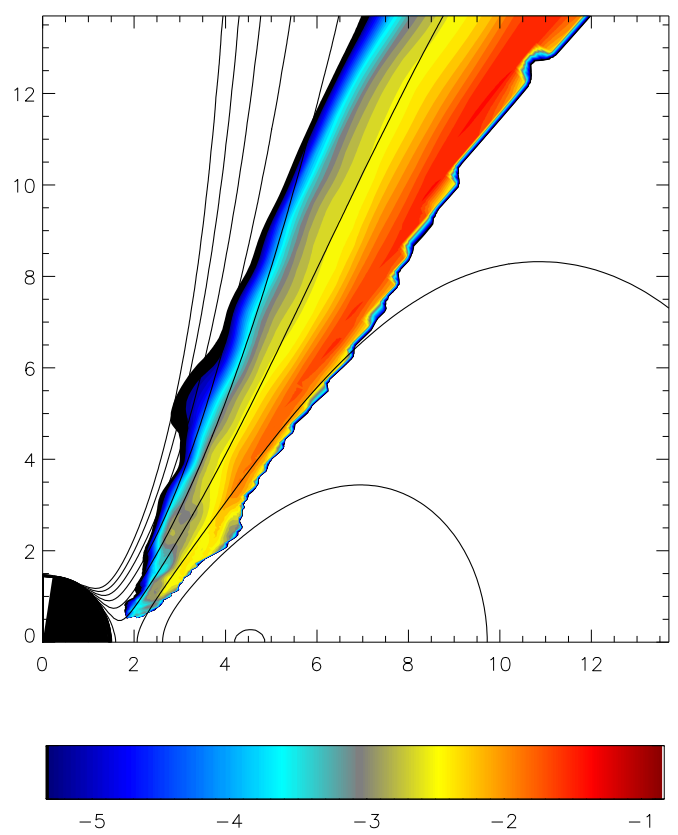

Figure 8: Instantaneous azimuthally-averaged radial momentum flux $\rho u^{r}$ (colored logarithmic contours) superimposed on contours (black curves) of the effective potential, all from a simulation with $a_{*}=0.9[8]$.

physics.

\section{Conclusions}

Although there is plenty left to do in the numerical study of accretion onto black holes and the associated jets, we have already arrived at a number of answers (or at least partial answers) to some of the questions raised at the beginning of this talk.

We have learned, for example, that large-scale fields can be created spontaneously from much smaller-scale fields, provided the initial field geometry is suitable. Radial when the black hole has no spin, the frame-dragging of rotating black holes twists magnetic fieldlines into helices around the rotation axis when the black hole spins. These large-scale fields can easily be strong enough to power a very substantial Poynting flux jet.

The power for the outflows appears to come from both the black hole's rotation and the accretion flow. The former is the primary supplier to the electromagnetic part, the latter is the main energy source to the matter part. Limitations in contemporary simulation codes make estimates of the power in the matter-dominated part much more uncertain than estimates of the electromagnetic luminosity.

Lastly, we have learned that in terms of its principal elements, the magnetically-dominated funnel jet is closely related to the dynamics of the Blandford-Znajek model: it is driven by a combination of poloidal magnetic fields and black hole rotation. On the other hand, at least in the conditions explored in the simulations so far, the matter-dominated part of the outflow is related to neither the Blandford-Znajek model nor the Blandford-Payne model: it is best thought of as a 
pressure-driven wind channeled by the equipotential surfaces formed by the black hole's gravity and the angular momentum of matter in the inner portion of the accretion flow.

\section{References}

[1] S.A. Balbus and J.F. Hawley 1998, Rev. Mod. Phys., 70, 1

[2] R.D. Blandford and R. Znajek 1977, MNRAS, 179, 433

[3] R.D. Blandford and D. Payne 1981, MNRAS, 199, 833

[4] J.-P. De Villiers and J.F. Hawley 2003, ApJ, 589, 458

[5] R.T. Emmering, R.D. Blandford and I. Shlosman 1992, ApJ, 385,460

[6] C.F. Gammie, J.C. McKinney and G. Tôth 2003, ApJ, 589, 444

[7] J. Gracia, K. Tsinganos and S.V. Bogovalov 2005, A\&A, 442, L7

[8] J.F. Hawley and J.H. Krolik 2006, ApJ, 641, 103

[9] S. Hirose, J.H. Krolik, J.-P. De Villiers, and J.F. Hawley 2004, ApJ, 606, 1083

[10] S.S. Komissarov 2004, MNRAS, 350, 1431

[11] D. Lynden-Bell 2003, 2003, 341, 1360

[12] J. McKinney and C.F. Gammie 2004, ApJ, 611, 977

[13] Y. Mizuno, K.-I. Nishikawa, S. Koide, P. Hardee and G.J. Fishman 2006, astro-ph/0609004

[14] M. Nakamura, H. Li and S.-T. Li 2006, ApJ, 652, 1059

[15] E.S. Phinney 2003, unpublished Cambridge University Ph.D. thesis

[16] M. Livio, G. Ogilvie, and J. Pringle 1999, ApJLett, 512, 100

[17] K.S. Thorne, R.H. Price and D.A. MacDonald 1986, Black Holes: The Membrane Paradigm, Yale University Press, New Haven

[18] D.A. Uzdensky and A. MacFadyen 2006, Apj, 647, 1192

[19] N. Vlahakis and A. Königl 2003, ApJ, 596, 1104 